\title{
Correlation between Circulating Tumor Cells and Plasma D-dimer and Clinicopathological Characteristics of Patients with Non-small Cell Lung Cancer
}

\author{
Junfu Wang ${ }^{1}$, Haibo Liư ${ }^{2}$ Xiaoming Sun ${ }^{2}$, Qiaoling Liu' ${ }^{2}$, Shuyi Han ${ }^{3}$ and Liangming Zhu ${ }^{4}$ \\ ${ }^{1}$ Department of Thoracic Surgery, School of Medicine, Weifang Medical University, Jinan Central Hospital Affiliated to Shandong \\ University, Shandong, China \\ ${ }^{2}$ Department of Thoracic Surgery, Jinan Central Hospital Affiliated to Shandong University, Jinan, Shandong, China \\ ${ }^{3}$ Department of Central Laboratory, Jinan Central Hospital Affiliated to Shandong University, Jinan, Shandong, China \\ ${ }^{4}$ Department of Thoracic Surgery, School of Medicine, Shandong University, Jinan Central Hospital Affiliated to Shandong University, \\ Jinan, Shandong, China
}

\begin{abstract}
Objective: To study the relationship between circulating tumor cells (CTCS) and plasma D-dimer (D-D) and their correlation with clinicopathological characteristics of patients with non-small cell lung cancer (NSCLC).

Study Design: A descriptive study.

Place and Duration of Study: Department of Thoracic Surgery, Jinan Central Hospital Affiliated to Shandong University, Jinan, Shandong, China, from December 2015 to August 2019.

Methodology: Seventy-five patients with NSCLC were selected as the research subjects. The contents of CTCS and D-D were determined and the results were analysed as per objective. Data was analysed quantitatively, using SPSS Version 24.0.

Results: Forty-five patients with NSCLC were positive for CTCS. The level of D-D in NSCLC patients was significantly increased. The D-D level in CTCs-positive patients was $0.79(0.43-1.80) \mathrm{mg} / \mathrm{L}$. The levels of CTCs and D-D in NSCLC patients were not affected by gender and pathological subtypes and other factors $(p>0.05)$. CTCs and D-D in peripheral blood of NSCLC patients were significantly correlated with the total stage of lung cancer patients $(p<0.05)$.

Conclusion: The levels of CTCS and D-D in peripheral blood of NSCLC patients are significantly correlated with clinicopathological features, and the positive CTCS and high levels of D-D may indicate the late stage of the disease and poor prognosis, and there is a correlation between the two. The combined detection of the two is of great significance in predicting the progress and poor prognosis of NSCLC patients, and can guide the clinical diagnosis and treatment.
\end{abstract}

Key Words: Non-small cell lung cancer (NSCLC), Circulating tumor cells (CTCS), D-dimer (D-D), Clinicopathologic features.

How to cite this article: Wang J, Liu H, Sun X, Liu Q, Han S, Zhu L. Correlation between Circulating Tumor Cells and Plasma D-dimer and Clinicopathological Characteristics of Patients with Non-small Cell Lung Cancer. J Coll Physicians Surg Pak 2021; 31(04):417-421.

\section{INTRODUCTION}

Lung carcinoma, also known as primary bronchogenic carcinoma, is one of the most common malignant tumors. It is common in men over the age of 40 years. In recent years, the incidence rate and mortality rate has increased significantly, which poses a major threat to human health and life. According to their biological characteristics, it is mainly divided into NSCLC and SCLC. NSCLC accounted for about $80 \%$ of all lung cancers, and the prognosis of patients is poor. ${ }^{1}$ Recurrence and metastasis often lead to death.

Correspondence to: Dr. Liangming Zhu, Department of Thoracic Surgery, Jinan Central Hospital Affiliated to Shandong University, No.105 Jiefang Road, Jinan, Shandong, China

E-mail: pengchongsd@sina.com

Received: October 31, 2020; Revised: March 10, 2021;

Accepted: March 27, 2021

DOI: https://doi.org/10.29271/jcpsp.2021.04.417
CTCs are the premise of distant metastasis of lung cancer and the key link of forming metastasis. ${ }^{2}$ Early detection of CTCs in peripheral blood of patients can indicate tumor metastasis. Most of the data published to date, including the results of almost all the clusters, strongly support the value of CTCS count as a survival predictor. ${ }^{3}$

Tumor can induce coagulation, and activated clotting substances can promote tumor growth, invasion, metastasis and angiogenesis. ${ }^{4}$ A number of studies have shown that changes in coagulation system function are significantly associated with tumor cell metastasis. ${ }^{5}$ D-D has been reported as a prognostic markerformany malignant tumors.

This study was conceived to analyse the correlation between the levels of CTCS and D-D in peripheral blood of patients with NSCLC and their clinicopathological characteristics, so as to provide reference for the diagnosis and treatment and prognosis of NSCLC patients, and better guide the clinical diagnosis and treatment. 
The purpose of this study was to investigate the relationship between CTCS and D-D in peripheral blood of patients with NSCLC and their relationship with clinicopathological features.

\section{METHODOLOGY}

Seventy-five NSCLC patients (Lung Cancer Group) from December 2015 to August 2019 in Jinan Central Hospital Affiliated to Shandong University were selected as the research subjects. Inclusion criteria were NSCLC patients diagnosed pathologically; aged 40-80 years; anti-tumor treatment-naive for any radiotherapy, chemotherapy and immunotheraphy, absence of thrombosis, coagulation system abnormalities, and willing to participate in the study. Exclusion criteria were history of chemoradiotherapy and anticoagulant or anti-platelet aggregation therapy, pregnancy, age less than 40 and over 80 years, primary malignant tumor in other organs; and anemia and severe heart, lung, liver, kidney and other diseases requiring treatment.

The study method was to collect $7.5 \mathrm{ml}$ venous blood from the patients. Blood samples should be detected within two hours and run abnormal cell separation staining apparatus detected. ${ }^{6}$ According to the operation and result interpretation of laboratory instruments (YZY Biopharma D100), the count of CTCS in peripheral blood $\geq 1 / 7.5 \mathrm{ml}$ was positive. The results of $D$-D were confirmed by coagulation function examination after admission. D-D content of samples was determined by monoclonal antibody immune projection turbidimetric method, which was completed within four hours.

SPSS version 24.0 software was used for statistical processing. The Shapiro-Wilk test was used to assess the normality of quantitative data. The categorical data were expressed as frequency and percentage; while quantitative data were expressed as median (IQR: Q1-Q3). The Chi-square test was used for inter-group comparison, and Mann-Whitney U-test was used for comparison. The difference was statistically significant $(p<0.05)$.

\section{RESULTS}

Among the 75 NSCLC patients, 45 were CTCs positive and 30 were CTCs negative (Figure 1). The level of D-D in CTC positive group was significantly higher than that in the negative group ( $p$ $<0.05$ ). The positive rate of CTC was $67.4 \%$ among the patients, whose D-D content exceeding the normal level, which suggesting that CTC may be associated with D-D. Details are presented in Tablel.

The results showed that 45 patients' CTC were positive and 30 were negative. According to the pathological subtypes, the expression levels of CTCs in patients with different pathological subtypes were analysed. The CTCs positive rate was $62.5 \%$ in adenocarcinoma patients and $52.6 \%$ in squamous cell carcinoma patients. The positive rate of adenocarcinoma patients was higher than that of squamous cell carcinoma patients, but there was no significant difference ( $p=0.448)$. On the basis of gender, to analyse the expression levels of CTCs in different gender. The CTCs positive rate was $62.2 \%$ in males and $56.7 \%$ in females. There was no significant difference $(p=0.630)$. The expression levels of CTCs in patients with different smoking conditions were analysed, according to smoking status. The CTCs positive rate was $65.5 \%$ in smokers and $56.5 \%$ in nonsmokers, there was no significant difference $(p=0.439)$. The expression levels of CTCs in patients with different stages were analysed by total stages. Total stage I II, III IV CTCs positive rate were $43.3 \%, 71.1 \%$, III IV period CTCs positive rate was significantly higher than that of $\mathrm{I}\|\|$ period $(\mathrm{P}=0.016)$. In addition, there was no significant correlation between CTCs and age ( $p>0.99)$. Details are presented in Table II.

Results analysis showed that there was no statistical significance between gender, pathological subtype and D-D level of NSCLC patients $(p>0.05)$. The $D-D$ 's level content of total stage I $\|$ and III IV was $0.38(0.20-0.73 \mathrm{mg} / \mathrm{L})$ and $0.85(0.54-1.80 \mathrm{mg} / \mathrm{L})$, respectively; while the level of stage III IV was significantly higher than stage $I \sim \|(p=0.001)$. In addition, D-D level was significantly different in various age and smoking status ( $p$ $<0.05$ ) (Table III).

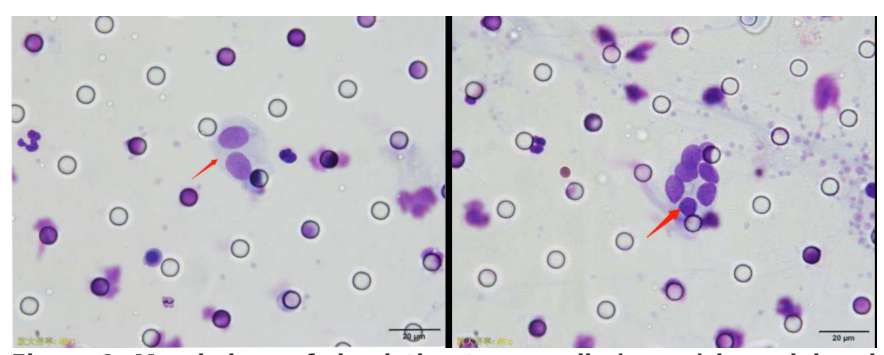

Figure 1: Morphology of circulating tumor cells (arrow) in peripheral blood of NSCLC patients $\times 50$.

Table I: The level of D-D in CTCs positive and negative groups.

\begin{tabular}{|l|c|c|}
\hline Group & $\mathbf{n}$ & D-D $\mathbf{~} \mathbf{m g} / \mathbf{L}$ \\
\hline CTCs positive & 45 & $0.79(0.43-1.80)$ \\
\hline CTCS negative & 30 & $0.49(0.22-0.84)$ \\
\hline$p$ & & 0.039 \\
\hline
\end{tabular}

\section{DISCUSSION}

Lung cancer is the most common malignant tumor in the world, and also the main cause of cancer death. ${ }^{7}$ Most of them have metastasis at the time of diagnosis, often losing the opportunity of surgical treatment, and the high incidence of local and distant metastasis lead to poor 5-year survival rate. ${ }^{8}$ Despite advances in surgical techniques, the 5-year survival rate of NSCLC patients treated surgically ranges from $25 \%$ to $73 \%$, depending on the disease stage. ${ }^{9}$ The traditional detection methods of lung cancer are limited because of their low sensitivity and poor tolerance. CTCs are the key link in the occurrence of distant metastasis, and have the advantages of easy access to specimens, small trauma and repeated use. Therefore, CTCs have become a gold indicator for monitoring tumor recurrence, guiding chemotherapy regimen and evaluating prognosis. ${ }^{10}$

Previous studies have found that the related factors of coagulation system may be related to the occurrence, development and metastasis of tumor. D-D is a sensitive index of hypercoagulability, which can reflect the activation level of coagulation and fibrinolysis. ${ }^{11}$ 


\begin{tabular}{|c|c|c|c|c|}
\hline Features & n (\%) & CTCs positive & CTCs negative & p-value \\
\hline \multicolumn{5}{|l|}{ Gender n (\%) } \\
\hline Male & 45 & $28(62.2)$ & $17(37.8)$ & \multirow{2}{*}{0.630} \\
\hline Female & 30 & $17(56.7)$ & $13(43.3)$ & \\
\hline \multicolumn{5}{|l|}{ Age (years) n (\%) } \\
\hline$\geq 60$ & 55 & $33(60.0)$ & $22(40.0)$ & \multirow{2}{*}{$>0.999$} \\
\hline$<60$ & 20 & $12(60.0)$ & $8(40.0)$ & \\
\hline \multicolumn{5}{|l|}{ Smoking status n (\%) } \\
\hline Smoking & 29 & $19(65.5)$ & $10(34.5)$ & \multirow{2}{*}{0.439} \\
\hline Non-smoking & 46 & $26(56.5)$ & $20(43.5)$ & \\
\hline \multicolumn{5}{|l|}{ Pathological subtype $\mathrm{n}(\%)$} \\
\hline Adenocarcinoma & 56 & $35(62.5)$ & $21(37.5)$ & \multirow{2}{*}{0.448} \\
\hline Squamous cell carcinomas & 19 & $10(52.6)$ & $9(47.4)$ & \\
\hline \multicolumn{5}{|l|}{ Total installment n (\%) } \\
\hline$I \sim \|$ & 30 & $13(43.3)$ & $17(56.7)$ & \multirow{2}{*}{0.016} \\
\hline IIII IV & 45 & $32(71.1)$ & $13(28.9)$ & \\
\hline
\end{tabular}

\begin{tabular}{|c|c|c|c|}
\hline Features & $\mathbf{n}$ & D-D (mg/L) & $p$-value \\
\hline \multicolumn{4}{|l|}{ Gender } \\
\hline Male & 45 & $0.69(0.45-1.80)$ & \multirow{2}{*}{0.096} \\
\hline Female & 30 & $0.46(0.21-1.07)$ & \\
\hline \multicolumn{4}{|l|}{ Age (years) } \\
\hline$\geq 60$ & 55 & $0.81(0.47-1.42)$ & \multirow[b]{2}{*}{0.003} \\
\hline$<60$ & 20 & $0.25(0.18-0.65)$ & \\
\hline \multicolumn{4}{|l|}{ Smoking status } \\
\hline Smoking & 29 & $0.79(0.49-2.10)$ & \multirow{2}{*}{0.017} \\
\hline Non-smoking & 46 & $0.53(0.21-1.05)$ & \\
\hline \multicolumn{4}{|l|}{ Pathological subtype } \\
\hline Adenocarcinoma & 56 & $0.58(0.24-1.11)$ & \multirow{2}{*}{0.106} \\
\hline Squamous cell carcinomas & 19 & $0.79(0.51-2.09)$ & \\
\hline \multicolumn{4}{|l|}{ Total installment } \\
\hline $\mathrm{I} \sim \mathrm{II}$ & 30 & $0.38(0.20-0.73)$ & \multirow{2}{*}{0.001} \\
\hline $\mathrm{III} \sim \mathrm{IV}$ & 45 & $0.85(0.54-1.80)$ & \\
\hline
\end{tabular}

In this study, the relationship between CTCS and D-D in NSCLC patients was studied. It was found that the level of D$\mathrm{D}$ in CTCs positive group was $0.79(0.43-1.80) \mathrm{mg} / \mathrm{L}$, which was significantly higher than that in CTCS negative group. This study suggests that there is a certain correlation between CTCS and D-D in NSCLC patients. Other studies have also shown that the hypercoagulable state of blood makes tumor cells more likely to adhere to the vascular wall of target organs and promote the formation of metastasis. ${ }^{12}$ At present, there are few clinical studies on the relationship between CTCS and D-D (coagulation-related factor) in NSCLC patients, and the interaction and influence between them need further and long-term clinical statistical analysis.

In this study, when analysing the correlation between CTCS,D-D and clinicopathological characteristics of the patients, it was found that there was no significant difference between the positive rate of CTCs and pathological subtypes $(p>0.05)$. The traditional theory holds that adenocarcinoma is more likely to spread into the blood and lymphatic system. ${ }^{13,14}$ This may be due to the different number and composition of research samples included in this study, resulting in differences between different research results. In addition, there was no significant difference in the positive rate of CTCS and D-D content in patients with NSCLC when compared with those in stage I, II, III and IV ( $p>0.05)$. It may be due to the limitation of sample size and other factors in this study that there may be bias. A large number of sample size studies are needed to evaluate the relationship between the positive rate of CTCS and D-D content among NSCLC patients. However, the positive rate of CTCs and content of D-D in stage I II were significantly lower than those in stage $\mathbb{I I} \sim \mathbb{N}(p<0.05)$. It is suggested that the levels of CTCS and D-D may increase in the early stage of NSCLC, and the positive rate of CTCS and the content of D-D gradually increase with the progression of the disease. The later the clinical stage, the higher the positive rate of CTCS and the content of D-D. In 2007, ASCO included CTCs as tumor markers. Although it has not been confirmed that CTCs can be used as a standard test to determine the stage, it is helpful to reflect the degree of tumor progression, so that the application of CTCs detection in clinical practice has become an effective supplement to the traditional TNM staging system, thus guiding the next step of treatment. Tumor progression is a complex process involving changes 
in different physiological systems. Studies have shown that activated coagulation in human cancer is related to angiogenesis, and the activation of coagulation in perivascular area can promote the formation of tumor matrix. ${ }^{15}$

The functional status of patients can also affect the coagulation system. The better the functional status is, the better the health status is. Patients with poor functional status often limit their movement or even stay in bed, increasing the risk of hypercoagulability. ${ }^{16}$ The results showed that with the increase of age, the level of D-D also increased significantly $(p=0.003)$, which may be due to the gradual increase of age, the gradual deterioration of functional status, and the corresponding gradual hypercoagulability of blood. Previous studies have shown that age has no significant predictive effect on D-D. ${ }^{17}$ Analysis of the reasons for this result may be due to the limitations of the selection of patients in this study, which may lead to the deviation of the research results. In future, it is necessary to increase the sample size to expand the representativeness of the subjects and further verify the research results. In addition, smoking can make the blood relatively hypercoagulable. The higher the smoking index is, the more obvious the hypercoagulable state is; and the easier it is to form thrombus. ${ }^{18}$ The results showed that the level of $D-D$ in smokers was significantly higher than that in nonsmokers $(p=0.017)$.

In NSCLC patients, high D-D level indicates low survival rate and early recurrence. ${ }^{19}$ Routine monitoring of D-D level in NSCLC surgery patients can be used as a reference for daily operation..$^{20}$ In addition, the meta-analysis of Wang et al. showed that the number of CTCS was positively correlated with clinicopathological stage of patients, and was also significantly related to lymph node metastasis. ${ }^{21}$ LV et al. used cell search system to detect CTCs in pulmonary venous blood of 32 patients with NSCLC. ${ }^{22}$ The results showed that the positive rate was $90.6 \%$ (29/32), and the number of CTCs was positively correlated with the tumor size $(p=0.012)$, and it showed that the detection of CTCS was helpful to early detection of lung cancer and possible micrometastasis. Tong proposed the detection method of CTCs in lung cancer, which provides the hope of real-time liquid biopsy for prognosis evaluation and treatment intervention of lung cancer ${ }^{23}$ Jiang et al. reported that preoperative D-D positive $(>0.55 \mathrm{mg} / \mathrm{l})$ was an important independent poor predictor of disease-free survival in patients with NSCLC. ${ }^{17}$ Taguchi et al. determined the plasma D-D level in 70 patients with lung cancer, and determined that low $D-D$ concentration predicted a longer survival time. ${ }^{24}$ The results of this study also confirm it. The combined detection of CTCS and D-D level in peripheral blood of patients can predict the clinicopathological features such as patient stage and prognosis assessment.

\section{CONCLUSION}

The levels of CTCS and D-D in peripheral blood of NSCLC patients are significantly correlated with clinicopathological features, and there is a correlation between them. Hypercoagulability state promotes tumor progression and metastasis. Preventive anticoagulant therapy may delay the progression and metastasis of cancer patients. Therefore, the combined detection of the two is of great significance in predicting the progress and poor prognosis of NSCLC patients, and can guide the clinical diagnosis and treatment.

\section{ETHICAL APPROVAL:}

This study was conducted after approval from the Institutional Review Board.

\section{CONFLICT OF INTEREST:}

The authors declared no conflict of interest.

\section{FUNDING:}

Thanks YZY Biopharma for funding this research experiment.

\section{PATIENTS' CONSENT:}

Informed consents were obtained from all the patients for performing all the tests and to publish the obtained data.

\section{AUTHORS' CONTRIBUTION:}

JW: Research design and conception, data analysis and manuscript writing.

HL: Result interpretation and discussion and supervised study.

$\mathrm{XS}, \mathrm{QL}, \mathrm{SH}$ : Literature search, data collection and revalidated data.

LZ: Final manuscript preparation, proofreading and final approval.

\section{REFERENCES}

1. Muineloromay L, Vieito M, Abalo A, Nocelo M, Barón, Francisco. Evaluation of circulating tumor cells and related events as prognostic factors and surrogate biomarkers in advanced nsclc patients receiving first-line systemic treatment. Cancers 2014; 6(1):153-65. doi: 10.3390/cancers6010153.

2. Liu Chunhua, Gong Ping, Yang Jie. Relationship between circulating tumor cells and coagulation factors in primary lung cancer patients. Chinese J Oncol 2016; 38(5):368-371. doi: 10.3760/cma.j.issn.0253-3766.2016.05.009.

3. Syrigos K, Fiste O, Charpidou A, Grapsa D. Circulating tumor cells count as a predictor of survival in lung cancer. Critical Reviews in Oncology/Hematology 2018; 125:60-8. doi: 10.1016/j.critrevonc.2018.03.004.

4. Nicole MK, Thomas LO, Charles WF. Impact of venous thromboembolism and anticoagulation on cancer and cancer survival. J Clinical Oncology 2009; 27(29):4902-11. doi: 10.1200/JCO.2009.22.4584.

5. Zhang XH, Zuo XR, Li YQ, Ma LX. The clinical significance of serum C-reactive protein in TNM staging of lung cancer and its application value in combination with fibrinogen and Ddimer for the evaluation of chemotherapy. Chinese J Gerontol 2015; (21):6135-6.

6. Li H, Song P, Zou B, Liu M, Cui K, Zhou P, et al. Circulating tumor cell analyses in patients with esophageal squamous 
cell carcinoma using epithelial marker-dependent and -independent approaches. Medicine 2015; 94(38):1565. doi: 10.1097/MD.0000000000001565.

7. Bray F, Ferlay J, Soerjomataram I, Siegel RL, Torre LA, Jemal A. Global cancer statistics 2018: globocan estimates of incidence and mortality worldwide for 36 cancers in 185 countries. CA: A Cancer Journal for Clinicians 2018; 68(6): 394-424. doi: 10.3322/caac.21492.

8. Siegel RL, Miller KD, Jemal A. Cancer statistics. CA: A Cancer J Clinicians 2015; 65(1):5-29. Doi: 10.3322/caac.21254.

9. Goldstraw P, Franzcr DB, Jett JR, Chevalier TL, Lim FRCS E, Nicholson FRCPATH AG, et al. Non-small-cell lung cancer. Lancet 2011; 378(9804):1727-40. doi.org/10.1016/ S0140-6736(10)62101-0.

10. Neki K, Kawahara H, Watanabe K, Toyama Y, Akiba T, Yanaga K. Usefulness of circulating tumor cells after preliminary chemotherapy for prediction of response to further anticancer therapy in patient-s with initially unresectable metastatic colorectal cancer. Anticancer Research 2013; 33(4):1769-72.

11. Ge LP, Li J, Bao QL, Chen P, Jiang Q, Zhu LR. Prognostic and predictive value of plasma d-dimer in advanced non-small cell lung cancer patients undergoing first-line chemotherapy. Clinical Translational Oncol 2015; 17(1):57-64. doi: 10.1007/s12094-014-1198-2.

12. Qiao L, Wang L, Lou J. Clinical value of circulating tumor cell in the differential diagnosis of solitary pulmonary nodule. Chinese J Laboratory Medicine 2016; 39(12):941-5.

13. Ren B, Wei X, Zou G, He J, Xu G, Xu F, et al. Cancer testis antigen SPAG9 is a promising marker for the diagnosis and treatment of lung cancer. Oncol Rep 2016; 35(5):2599-05. doi: 10.3892/or.2016.4645.

14. Huang B, Zhou H, Wang S, Lang XP, Wang X. Effect of silencing SATB1 on proliferation, invasion and apoptosis of A549 human lung adenocarcinoma cells. Oncol Lett 2016; 12(5): 3818-24. doi: 10.3892/ol.2016.5179.

15. Tas F, Kilic L, Duranyildiz D. Coagulation tests show significant differences in patients with breast cancer. Tumor Biology 2014; 35(6):5985-92. doi: 10.1007/s13277-
014-1793-4.

16. Xu CH, Yu LK, Zhang Y, Xie HY, Hao KK, Hu W, et al. Analysis of prognostic factors of non-small cell lung cancer in patients under 40 years of age. Chinese J Oncol 2012; 34(9):703-5. doi: 10.3760/cma.j.issn.0253-3766. 2012.09.013.

17. Jiang HG, Li J, Shi SB, Chen P, Ge LP, Jiang Q, et al. Value of fibrinogen and d-dimer in predicting recurrence and metastasis after radical surgery for non-small cell lung cancer. Med Oncol 2014; 31(7):22-50. doi: 10.1007/s12032014-0022-8.

18. Zhou Yan. Relationship between smoking indexes and coagulation function in patients with lung cancer. Chinese J Clinical Oncol Rehabilitation 2016; (10):1160-2.

19. Shiina Y, Nakajima T, Yamamoto T, Tanaka K, Sakairi Y, Wada $\mathrm{H}$, et al. The D-dimer level predicts the postoperative prognosis in patients with non-small cell lung cancer. PloS One 2019; 14(12):e0222050. doi: 10.1371/journal.pone. 0222050 .

20. Deng HY, Zheng X, Jiang R, Wang RL, Zhou J, Qiu XM. Preoperative $D$-dimer level is an independent prognostic factor for non-small cell lung cancer after surgical resection: A systematic review and meta-analysis. Annals Translational Medicine 2019; 7(16):366. doi: 10.21037/atm.2019.05.35.

21. Wang J, Wang K, Xu J, Huang J, Zhang T. Prognostic significance of circulating tumor cells in non-small-cell lung cancer patients: A meta-analysis. Plos One 2013; 8(11):e78070. doi: 10.1371/journal.pone.0078070.

22. Lv C, Zhao B, Wang L, Zhang P, Ma Y, Wang Y, et al. Detectionof circulating tumor cells in pulmonary venous blood for resectable non-small cell lung cancer. Oncol Lett 2018; 15(1):1103-1112. doi: 10.3892/ol.2017.7405.

23. Tong B. Circulating tumor cells in patients with lung cancer: developments and applications for precision medicine. Future Oncol 2019; 15(21):2531-42. doi: 10.2217/fon-2018-0548.

24. Taguchi O, Gabazza EC, Yasui H, Kobayashi T, Yoshida M, Kobayashi H. Prognostic significance of plasma D-dimer levels in patients with lung cancer. Thorax 1997; 52(6):563-5. doi: 10.1136/thx.52.6.563. 